\title{
SAXS Study on Plasticized Poly(methyl methacrylate) Ionomers
}

\author{
Joon-Seop $\mathrm{KIM}^{\dagger}$ and Adi EISENBERG* \\ Department of Polymer Science \& Engineering, Chosun University, Kwangju 501-759, Korea \\ * Department of Chemistry, McGill University, Montreal, Quebec H3A 2K6, Canada
}

(Received March 30, 1998)

\begin{abstract}
KEY WORDS Ionomer / Small-Angle X-Ray Scattering / Plasticization / Poly(methyl methacrylate) / Cluster/Morphology /
\end{abstract}

Ionomers are polymers containing small amounts of ionic groups. ${ }^{1}$ Unique features of ionomers are the presence of a small-angle X-ray scattering (SAXS) peak and two phase behavior found in the dynamic mechanical studies of ionomers. According to a recent model of the morphology of random ionomers, the so-called EHM model, ${ }^{2}$ ionic groups form ionic aggregates, i.e., multiplets. ${ }^{3}$ The polymer chains surrounding the multiplets may be restricted in their mobility. ${ }^{2}$ As ion content increases, the number of multiplets increases, and regions of reduced mobility begin to overlap. When the size of the overlapping regions of restricted mobility, i.e., clusters, reaches dimensions of a $c a .100 \AA$ or larger, the material shows two glass transitions; one for the matrix regions and the other for cluster regions.

In general, clustered ionomer systems show SAXS peaks at characteristic $q(=4 \pi \sin \theta / \lambda$, where $\theta$ is half the scattering angle and $\lambda$ is an $\mathrm{X}$-ray wavelength). The position of the SAXS peak is associated with the intermultiplet distance. ${ }^{4,5}$ Another interpretation of the SAXS profile has also been proposed. ${ }^{6}$ The morphology and mechanical properties of unclustered ionomers are quite different from those of clustered ionomers. In the case of poly(methyl methacrylate-co-cesium methacrylate) (P(MMA-co-MACs)) ionomers, no SAXS peak was observed even for a sample containing $7.8 \mathrm{~mol} \%$ of ions. ${ }^{7}$ A single glass transition $\left(T_{\mathrm{g}}\right)$ was found dynamic mechanically for a $9.9 \mathrm{~mol}^{\%}$ sample. ${ }^{8}$ Thus it was concluded that the cesium neutralized PMMA ionomer is unclustered. The lack of clustering may be due to the high dielectric constant of the PMMA polymer, which weakens the interactions between ionic groups, and high $T_{\mathrm{g}}$ of the PMMA polymer, which disrupts multiplet formation. Very recently, we studied the plasticization of this ionomer system and found that plasticization induces cluster formation. ${ }^{9}$ When the ionomer was plasticized with 4-decylaniline, the ionomer exhibited a weak second $T_{\mathrm{g}}$ at higher temperature than the matrix $T_{\mathrm{g}}$. The effectiveness of the plasticization was ranked in the following order: glycerol $\approx 4$-decylaniline $>$ dioctyl phthalate.

The present study investigates the effects of plasticization on the morphology of the plasticized P(MMA-coMACs) ionomers and observes morphological changes of ionomers induced by the addition of three plasticizers; one polar plasticizer, i.e., glycerol (Gly), and two non-

\footnotetext{
† To whom all correspondence should be addressed.
}

polar plasticizers, i.e., dioctyl phthalate (DOP) and 4-decylaniline (4DA).

\section{EXPERIMENTAL}

\section{Plasticization}

A P(MMA-co-MACs) ionomer containing $9.4 \mathrm{~mol} \%$ of ionic groups was prepared as part of another study. ${ }^{8}$ To plasticize the ionomers, we dissolved the ionomer in a benzene-methanol solvent $(9: 1, \mathrm{v} / \mathrm{v})$ and added a plasticizer to the ionomer solution. Ionomer-plasticizer mixtures were freeze-dried and further dried under vacuum at $80^{\circ} \mathrm{C}$ for 1 day. The notation of the samples, and types and amounts of the plasticizers used are listed in Table I.

\section{Sample Preparation}

The samples were compression molded at $220^{\circ} \mathrm{C}$ under a pressure of $c a$. $20 \mathrm{MPa}$. The molded samples in the form of a thin disk with dimensions of $12 \mathrm{~mm}$. (diameter) $\times 0.6 \mathrm{~mm}$ (thickness) were stored under vacuum at $80^{\circ} \mathrm{C}$ for $24 \mathrm{~h}$.

\section{$S A X S$ Experiments}

SAXS experiments for the plasticized ionomers were conducted at D-22 station of the LURE-DCI synchrotron radiation source in Orsay, France. ${ }^{10} \mathrm{~A}$ fixed-exit, double-crystal monochromator was used to provide a beam of $9000 \mathrm{eV} \mathrm{X}$-rays $(\lambda=1.377 \AA)$. The size of the beam at the sample was smaller than $2 \mathrm{~mm}^{2}$. The detectors, consisting of two beam deflectors and a $\mathrm{NaI}$ scintillator, were positioned before and after a sample chamber to monitor the relative input of the X-ray intensity and sample absorption. A one-dimensional position-sensitive detector, filled with $\mathrm{Xe}-\mathrm{CO}_{2}$ gas, with

Table I. Sample notations and types and amounts of plasticizers

\begin{tabular}{ccc}
\hline & & \multicolumn{2}{c}{ Amount of plasticizer } \\
\cline { 3 - 3 } Sample notation & Type of plasticizer & wt $\%$ \\
\hline Gly-13\% & Glycerol & 12.6 \\
Gly-27\% & Glycerol & 26.8 \\
DOP-13\% & Dioctyl phthalate & 13.0 \\
DOP-26\% & Dioctyl phthalate & 26.4 \\
4DA-14\% & 4-Decylaniline & 13.7 \\
4DA-26\% & 4-Decylaniline & 26.0 \\
\hline
\end{tabular}




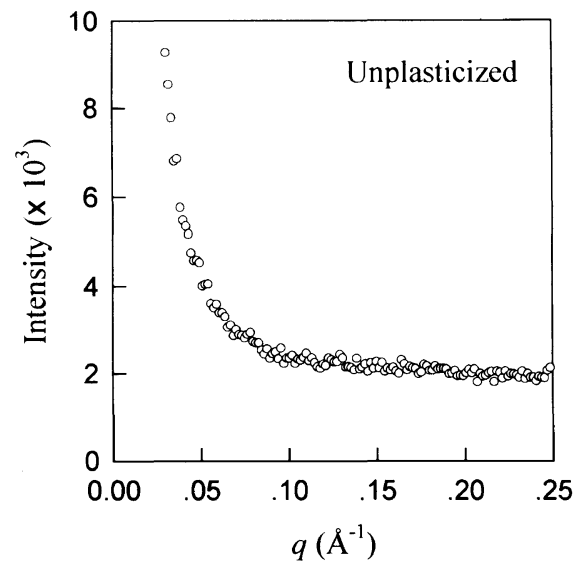

Figure 1. SAXS profile plotted as relative intensity $v s$. $q$ for unclustered Cs-neutralized PMMA ionomer containing $9.4 \mathrm{~mol} \%$ ions.

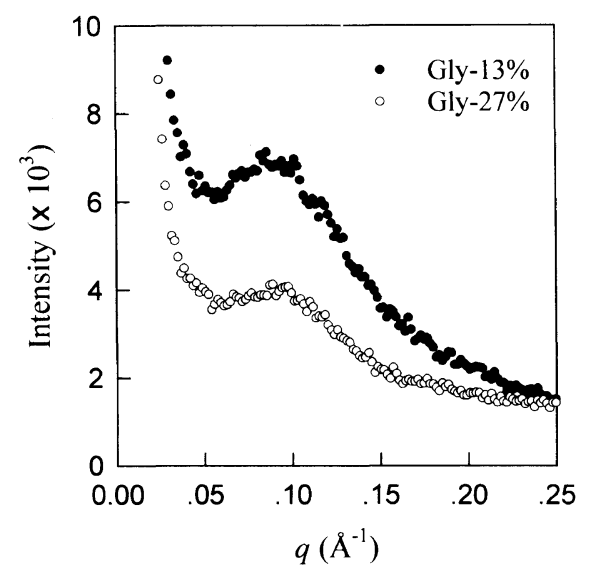

Figure 2. SAXS data for Gly-plasticized PMMA ionomers.

a resolution of $200 \mu \mathrm{m}$ was used to detect the scattered X-rays. A sample-to-detector distance of $570 \mathrm{~mm}$ allowed for SAXS data to be obtained in the $q$ range from $c a$. 0.012 to $0.32 \AA^{-1}$. All samples were exposed to the beam for $5 \mathrm{~min}$. SAXS data were plotted as relative intensity vs. $q$ after correction for background scattering. All samples were normalized to a thickness of $1 \mathrm{~mm}$.

\section{RESULTS AND DISCUSSION}

The SAXS profile for unplasticized P(MMA-coMACs) containing $9.4 \mathrm{~mol} \%$ of ions is shown in Figure 1. As found before for $4.7-7.8 \mathrm{~mol} \%$ samples, ${ }^{7}$ no SAXS peak was seen, only a small-angle upturn (SAUT) $(q<$ $\left.0.05 \AA^{-1}\right)$. We do not discuss further the basis of the SAUT since its interpretation has been subjected to some controversy. ${ }^{11-14}$ The absence of the peak suggests that in the ionomer scattering centers are not present in the range of Bragg distances between 20 and $500 \AA$.

In Figure 2, the results of the SAXS experiments on the Gly-plasticized ionomers are shown. SAXS peaks are seen for Gly-13\% and Gly-27\% samples at similar $q$ values of $c a \cdot 0.09 \AA^{-1}\left(d_{\mathrm{Bragg}}=c a\right.$. $\left.70 \AA\right)$. The relative intensity of the peak decreases with plasticizer content. The presence of plasticizer induces multiplet (i.e., ionic aggregate) formation. Since the Gly plasticizer resides in multiplets because of its high polarity, it increases the volume of the multiplets. As a result, the plasticizer 304

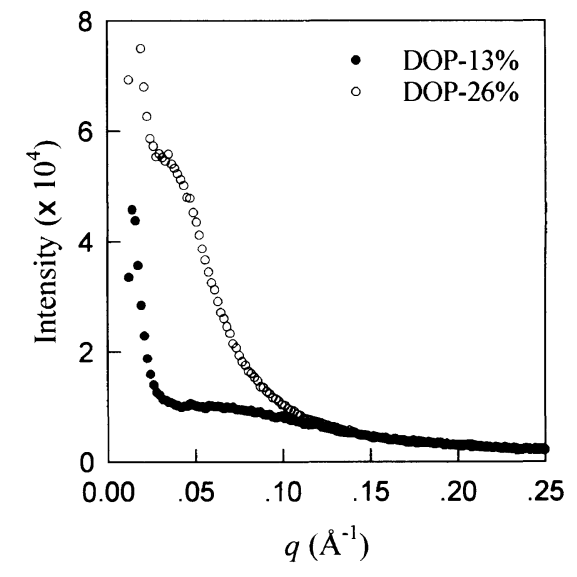

Figure 3. SAXS data for DOP-plasticized PMMA ionomers.

decreases electron density of the scattering centers. Thus, the scattering intensity decreases with plasticizer content. It should be mentioned that increase in the amount of the plasticizer from 13 to $27 \%$ enlarges the size of scattering centers diluted with Gly and, in turn, may alter the distance between scattering centers, but only slightly, since the scattering centers are located at threedimensional sites. Also, there appears what seems to be a weak second peak at $c a . q=0.17 \AA^{-1}$ for the Gly-27\% sample. Two explanations are possible; this feature may be due to the arrangement of scattering centers becoming probably more ordered with increase in amount of plasticizer, or due to simple fluctuation. However, at this stage we do not have any clear explanations.

The SAXS results on ionomers plasticized with DOP are shown in Figure 3. A very weak peak is shown for the DOP-13\% sample at a $q$ value $<c a .0 .05 \AA^{-1}$ $\left(d_{\text {Bragg }}>125 \AA\right)$. The intensity of the peak is higher than those for Gly-plasticized samples. For the DOP-26\% sample, a peak is seen as a shoulder of SAUT at smaller $q$. This suggests that scattering centers are developing with plasticizer content. However, distances between scattering centers are too large to be interpreted as intermultiplet distances but as distances between phaseseparated ionomer regions, i.e., inter-ionomer distances. Thus, as the amount of the plasticizer increases, the distances of average separation between ionomer regions increase, and the peak shifts to a smaller angle. With increase of the DOP content, the phase-separated ionomer regions start to aggregate, and thus the intensity of the SAXS peak increases.

SAXS results on the 4DA-plasticized ionomers are shown in Figure 4. A SAXS peak is seen for the 4DA-14\% sample at $q$ of $c a .0 .11 \AA^{-1}$ and $d_{\text {Bragg }}=c a .57 \AA$, and a very well developed peak for the $4 \mathrm{DA}-26 \%$ sample at $q=c a .0 .14 \AA^{-1}\left(d_{\mathrm{Bragg}}=c a .45 \AA\right)$. This suggests that more multiplets form with plasticizer content, which leads to increase in the number density of multiplets. The increase in the number density of scattering centers causes shifts of SAXS peaks to higher q, i.e., shorter Bragg spacing. It should be recalled that increase in the size of multiplets leads to increase in the intensity of SAXS profile. ${ }^{7}$ However, in this $4 \mathrm{DA}$ system, it is the number of multiplets, not the size, that is expected to increase. Thus, only shifts of SAXS peaks were observed. Even though the Bragg distance between multiplets becomes 


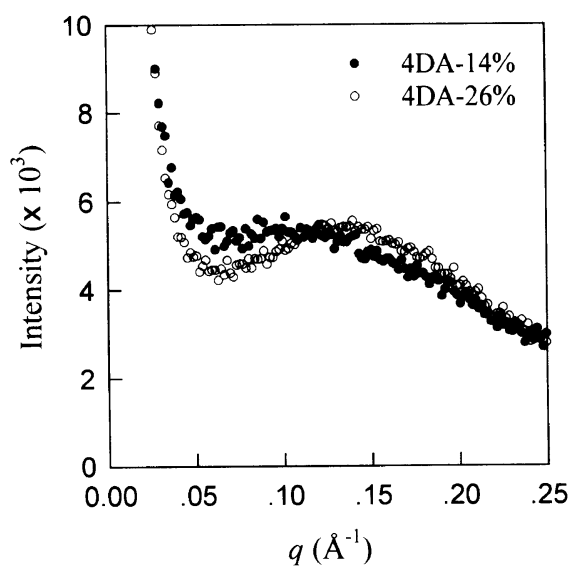

Figure 4. SAXS data for 4DA-plasticized PMMA ionomers.

shorter with plasticizer content, average Bragg distance of $c a .40 \AA$ is still too large for the PMMA ionomer to have a significant amount of the cluster phase. Therefore, only a small percentage of ionic groups may exist in the cluster region. Indeed, in a mechanical study of this system, a weakly developed $\tan \delta$ peak was seen at high temperature of the matrix $\tan \delta$ peak as a shoulder. ${ }^{9}$ From these morphological and dynamic mechanical findings, it is concluded that plasticization of the unclustered PMMA ionomer with 4DA molecules reduces the matrix $T_{\mathrm{g}}$ strongly, which makes electrostatic force relatively more important and gives the ionic groups enough energy to form multiplets. Thus, above a certain amount of the plasticizer, the proximity of multiplets is apparently sufficient for clustering to occur.
Acknowledgment. This work was partly supported by the Natural Sciences and Engineering Research Council of Canada (NSERC). The authors thank the reviewers for their valuable suggestions.

\section{REFERENCES}

1. (a) A. Eisenberg and M. King, "Ion-Containing Polymers, Physical Properties and Structure." Academic Press, New York, N.Y., 1977; (b) A. Eisenberg and J.-S. Kim, "Introduction to Ionomers," John Wiley \& Sons, New York, N.Y., 1998.

2. A. Eisenberg, B. Hird, and R. B. Moore, Macromolecules, 23, 4098 (1990).

3. A. Eisenberg, Macromolecules, 3, 147 (1970).

4. D. J. Yarusso and S. L. Cooper, Macromolecules, 16, 1871 (1983).

5. R. B. Moore, D. Bittencourt, M. Gauthier, C. E. Williams, and A. Eisenberg, Macromolecules, 24, 1376 (1991).

6. W. J. MacKnight, W. P. Taggert, and R. S. Stein, J. Polym. Sci., Polym. Symp., 45, 113 (1974).

7. M. Jiang, A. A. Gronowski, H. L. Yeager, G. Wu, J.-S. Kim, and A. Eisenberg, Macromolecules, 27, 6541 (1994).

8. A. A. Gronowski, M. Jiang, H. L. Yeager, G. Wu, and A Eisenberg, J. Membrane Sci., 82, 83 (1993).

9. J.-S. Kim, H.-S. Kim, and A. Eisenberg, Bull. Korean Chem. Soc., 19, 623 (1998).

10. J. M. Dubuisson, J. M. Dauvergne, C. Depautex, P. Vachette, and C. E. Williams, Nucl. Instrum. Methods Phys. Res., A246, 636 (1986).

11. C. E. Williams, T. P. Russell, R. Jérôme, and J. Horrion, Macromolecules, 19, 2877 (1986).

12. A. F. Galambos, W. B. Stocken, J. T. Koberstein, A. Sen, R. A. Weiss, and T. P. Russell, Macromolecules, 20, 3091 (1987).

13. Y. S. Ding, S. R. Hubbard, R. A. Register, and S. L. Cooper, Macromolecules, 21, 1698 (1988).

14. R. B. Moore, M. Gauthier, C. E. Williams, and A. Eisenberg, Macromolecules, 25, 5769 (1992). 